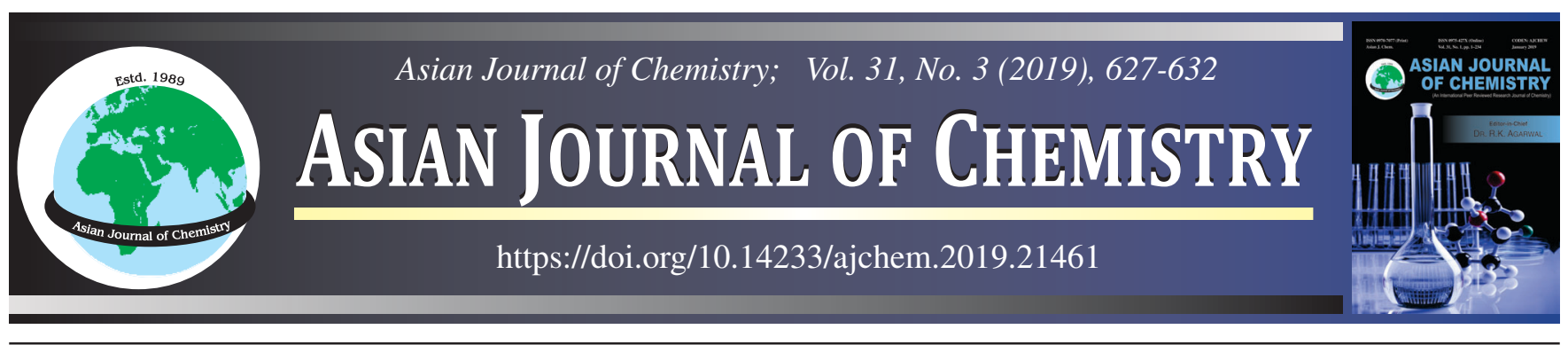

\title{
Synthesis and Antibacterial Evaluation of Hydrazone Derivatives Bearing 6-Chlorothieno[3,2-c]pyridine Moiety
}

N. Sree Lakshmana Rao ${ }^{1}$, Mandava V. Basaveswara RaO ${ }^{2, *}$ and K.R.S. Prasad ${ }^{1}$

${ }^{1}$ Department of Chemistry, Koneru Lakshmiah Education Foundation, Green Fields, Vaddeswaram-522502, India

${ }^{2}$ Department of Chemistry, Krishna University, Machilipatnam-521001, India

*Corresponding author: E-mail: mandavabasaveswararaov@gmail.com

Received: 19 May 2018;

Accepted: 21 November 2018;

Published online: 31 January 2019;

AJC-19258

The present investigation describes the overall preparation of 6-chlorothieno[3,2-c]pyridine-2-carbohydrazide-hydrazone subsidiaries (7a-r) from industrially accessible ethyl-4,6-dichloronicotinate as starting material. The synthesis of these derivatives involve some prominent reactions such as (i) $\mathrm{NaBH}_{4}$ reduction of ethyl ester group (ii) $\mathrm{MnO}_{2}$ oxidation of $1^{\circ}$ alcohol (iii) cyclization of aldehyde with ethylmercapto acetate leading to thieno[3,2-c]pyridine ring (iv) hydrazinolysis of the ethyl group (v) condensation of selected aldehydes with 6chlorothieno[3,2-c]pyridine-2-carbohydrazide leading to the desired 6-chlorothieno[3,2-c]pyridine-2-carbohydrazide-hydrazone derivatives (7a-r). The structure of the derivatives was characterized by elemental analysis, NMR, infrared and mass spectroscopic analysis. These compounds were screened for their antibacterial property towards Gram positive and Gram negative bacterial strains using agar diffusion method with reference to norfloxacin as reference antibiotic.

Keywords: Ethyl-4,6-dichloronicotinate, Carbohydrazide-hydrazone, Antibacterial activity, Thieno[3,2-c]pyridine.

\section{INTRODUCTION}

The drugs especially antibiotics related to microorganism infections presently characterize a considerable therapeutic task throughout the world. Although these antibiotics are utilized for many infectious, the influx of newest infectious diseases as well as additive levels of resistance in some pathogens-impend to weaken the success of the few remaining medication offered for the treatment of those infections $[1,2]$. The scarcity of innovation within the development of latest antibiotics has considerably inflated the challenge of treating and eliminating bound infecting pathogens, that cause a hour fall within the numbers of latest approvals and few novel molecules [2]. Whereas this trend is dynamic, there's an pressing demand for the event of latest medicinal drug agents with divergent and distinctive structural options that operate via a distinct mechanisms of action to those of existing antimicrobial agents [2,3].

In recent years, interest in one of the important hetrocyclic ring nuclei such as thieno[3,2-c]pyridines has emerged due to their similarity to benzofuran, quinoline and isoquinoline, which are important nuclei present in many biologically active comp- ounds. Thiophenes with fused six membered hetrocyclic ring are currently attracting special interest. In numerous therapeutic areas, thieno[3,2-c]pyridine derivatives was found to exhibit various medicinal properties viz., antithrombin activity [4], antipsychotic activity [5] and as a protease kinase inhibitor [6]. Furthermore, in recent years, some of thieno[3,2-c] derivatives exhibited fluorescence properties $[7,8]$.

Hydrazone derivatives have created a lot of widespread research in their utility and have been utilized as important precursors in the construction of various heterocyclic ring reactions [9]. Hydrazones constitute a class of organic compounds, which attracts the attention of medicinal chemists due to the fact that they contain azomethine group $(-\mathrm{NH}-\mathrm{N}=\mathrm{CH}-)$ connected with carbonyl group, which is responsible for their various biological activities [9], viz., anticancer [10], anti-inflammatory [11], antiviral [12]. In recent years, compounds with hydrazidehydrazone structure have been found to exhibit antibacterial activity to a significant level [13]. Certain complexes of hydrazones catalyze physiological processes so as to get transformed to go about as sterilants for houseflies, nematocides, herbicides, bug sprays, rodenticides and plant development controllers

This is an open access journal, and articles are distributed under the terms of the Creative Commons Attribution-NonCommercial-ShareAlike 4.0 (CC BY-NC-SA 4.0) International License which allows readers to freely read, download, copy, distribute, print, search, or link to the full texts of its articles and to use them for any other lawful non-commercial purpose as long as the original source is duly acknowledged. 
[14-16]. Furthermore, hydrazones discover applications in colorimetric or fluorimetric determinations, specifically, an expanding number of $\mathrm{N}-\mathrm{N}$ bond-containing heterocycles and peptidomimetics have advanced into commercial applications as pharmaceutical and horticultural agents [17-19].

Inspired with the above biological activities of hydrazidehydrazones and in continuation to previous research on thiophene$[3,2-c]$ pyridine and furo[3,2-c]pyridine hydrazide-hydrazone derivative [20,21], we report here in the preparation, structural determination and screening results of antibacterial activity of a new series of 6-chlorothieno[3,2-c]pyridine-2-carbohydrazidehydrazone derivatives (7a-r).

\section{EXPERIMENTAL}

Reagents and solvents obtained from commercial suppliers were used without purification or drying unless otherwise noted. ${ }^{1} \mathrm{H}$ NMR were recorded using Bruker \& Agilent instrument at $400 \mathrm{MHz}$ and TMS was used as an internal standard. LCMS analysis was performed on Waters UPLC with SQD-2 mass detector (single quadrupole). $\delta$ parts per million (ppm) a unit of measurement for chemical shifts are reported downfield from tetramethylsilane (TMS) with reference to internal solvent and coupling constants in Hz. The mass spectra were recorded on Agilent ion trap MS. Infrared spectra were recorded on a Perkin Elmer FT-IR spectrometer. TLC analysis was monitored used silica gel $60 \mathrm{~F}_{254}$ pre-coated plates prior to the use with appropriated eluents necessary for the experiments. Silica gel 60-120/100-200 mesh was used for column chromatography. Mel-temp apparatus was used for the determination of melting point and are uncorrected.

(4,6-Dichloropyridin-3-yl)methanol (2): In a mixture of tetrahydrofuran:methanol $(8: 2)(200 \mathrm{~mL})$ was added ethyl-4, 6-dichloronicotinate (1) (10.0 g, $45.45 \mathrm{mmol})$ followed by $\mathrm{NaBH}_{4}(3.45 \mathrm{~g}, 90.90 \mathrm{mmol})$ at $0{ }^{\circ} \mathrm{C}$. After $4 \mathrm{~h}$ of stirring at room temperature, the reaction mixture was quenched with water at $0{ }^{\circ} \mathrm{C}$ and extracted with ethyl acetate. After usual work up procedure, compound $\mathbf{2}$ was isolated. Yellow solid; Yield: $7.2 \mathrm{~g}, 82 \%$.Elemental analysis of $\mathrm{C}_{6} \mathrm{H}_{5} \mathrm{NOCl}_{2}$ calcd. (found) \%: C 40.48 (40.50); H 2.83 (2.80); N 7.87 (7.86).

4,6-Dichloronicotinaldehyde (3): $\mathrm{MnO}_{2}(21.23 \mathrm{~g}, 244$ $\mathrm{mmol}$ ) was added to a pre-mixed solution of compound 2 (7.2 g, $40.67 \mathrm{mmol})$ in methanol $(140 \mathrm{~mL})$. The reaction content was stirred and heated to $60{ }^{\circ} \mathrm{C}$ for $18 \mathrm{~h}$ and filtered while hot and the inorganic discharge was washed with methanol. The combined filtrate was concentrated to obtain compound 3. Yellow solid; Yield: $5 \mathrm{~g}, 70 \%$. Elemental analysis of $\mathrm{C}_{6} \mathrm{H}_{3} \mathrm{NOCl}_{2}$ calcd. (found) \%: C 40.95 (40.97); H 1.72 (1.70); N 7.96 (7.94).

Ethyl 6-chlorothieno[3,2-c]pyridine-2-carboxylate (4): A pre-mixed stirred solution of compound 3 (7.4 g, 42.28 $\mathrm{mmol})$ in dichloromethane $(40 \mathrm{~mL})$ containing triethyl amine (11.37 mL, $84.56 \mathrm{mmol}$ ) was added ethylmercapto acetate $(5.59 \mathrm{~mL}, 50.74 \mathrm{mmol})$ at $0^{\circ} \mathrm{C}$. The reaction mixture was stirred at room temperature for $1 \mathrm{~h}$ and then to $50{ }^{\circ} \mathrm{C}$ for $18 \mathrm{~h}$. The reaction mixture was quenched with water and extracted with ethyl acetate. After usual standard work up procedure and purification by column chromatography (silica gel: 100-200 mesh, eluent: $5 \%$ ethyl acetate in pet ether) isolated compound 4; Light Yellow solid; Yield: 5.7 g, 56 \%; m.p.: 108-112 ${ }^{\circ} \mathrm{C}$.
Elemental analysis of $\mathrm{C}_{10} \mathrm{H}_{8} \mathrm{NO}_{2} \mathrm{SCl}$ calcd. (found) \%: C 49.69 (49.71), H 3.34 (3.32), N 5.80 (5.77)

6-Chlorothieno[3,2-c]pyridine-2-carbohydrazide (5): Hydrazine hydrate monohydrate $(4.88 \mathrm{~mL}, 99.58 \mathrm{mmol})$ was added to a pre-mixed solution of compound 4 (3.0 g, 12.44 $\mathrm{mmol})$ in ethanol $(240 \mathrm{~mL})$. The reaction content was stirred at room temperature for $1 \mathrm{~h}$ and later heated to $80^{\circ} \mathrm{C}$ for $18 \mathrm{~h}$. The reaction mixture was evaporated under reduced pressure and the residue was triturated with $n$-pentane followed by methanol to result in compound 5. Off white solid; Yield: 2.5 g, $88 \%$ ); m.p.: $282-286{ }^{\circ} \mathrm{C}$; IR ( KBr, $\left.v_{\max }, \mathrm{cm}^{-1}\right): 3316\left(-\mathrm{NH}_{2}-\right)$, 1647 (-C=O), 1574 (-C=N str.), 1367 (C-N str.). ${ }^{1} \mathrm{H}$ NMR (500 MHz, DMSO- $\left.d_{6}\right): \delta 10.30$ (brs, $\left.1 \mathrm{H}\right), 9.10$ (s, $\left.1 \mathrm{H}\right), 8.30$ (s, 1H), 8.18 (s, 1H), 4.75 (brs, 2H). ${ }^{13} \mathrm{C}$ NMR (100 MHz, DMSO- $\left.d_{6}\right)$ : $\delta$ 160.40, 149.58, 147.02, 145.29, 141.19, 135.32, 121.89, 117.48; ESI MS: $m / z, 28.08(\mathrm{M}+\mathrm{H})^{+}$. Elemental analysis of $\mathrm{C}_{8} \mathrm{H}_{6} \mathrm{~N}_{3} \mathrm{OSCl}$ calcd. (found) \%: C 42.20 (42.18); $\mathrm{H} 2.66$ (2.68); N 18.46 (18.44).

Synthesis of 6-chlorothieno[3,2-c]pyridine-2-carbohydrazide-hydrazone derivatives (7a-r): Respective aromatic/ hetro aromatic aldehydes $(\mathbf{6 a - r})(0.43 \mathrm{mmol})$ was added to a pre-mixed solution of compound $5(0.43 \mathrm{mmol})$ in ethanol. The reaction content was refluxed for $0.5 \mathrm{~h}$. The solids was filtered and rinsed with ethanol and dried to afford the respective hydrazone derivatives (7a-r) in 82-93\% yield (Scheme-I).

$(E)$ - $N^{\prime}$-Benzylidene-6-chlorothieno[3,2-c]pyridine-2carbohydrazide (7a): White solid; Yield: $120 \mathrm{mg}, 86.5 \%$; m.p.: 275-279 ${ }^{\circ} \mathrm{C}$; IR (KBr, $\left.v_{\max }, \mathrm{cm}^{-1}\right)$ : 3228 (-CO-NH str.), 1638 (-C=O str.), 1558 (-C=N str.), 1364 (-C=C str.), $1263(-\mathrm{C}-\mathrm{N}$ str.), $636(\mathrm{C}-\mathrm{Cl}) .{ }^{1} \mathrm{H}$ NMR (500 MHz, DMSO-d $): \delta 12.32$ $(* 12.23, \mathrm{~s}, 1 \mathrm{H}), 9.16(\mathrm{~d}, J=9.5 \mathrm{~Hz}, 1 \mathrm{H}), 8.55(* 8.48, \mathrm{~s}, 1 \mathrm{H})$, $8.36(\mathrm{~d}, J=8.0 \mathrm{~Hz}, 2 \mathrm{H}), 8.19(\mathrm{~s}, 1 \mathrm{H}), 7.87(\mathrm{~d}, \mathrm{~J}=7.0 \mathrm{~Hz}, 1 \mathrm{H})$, $7.77(\mathrm{~d}, J=5.5 \mathrm{~Hz}, 1 \mathrm{H}), 7.54-7.48(\mathrm{~m}, 3 \mathrm{H})$; ESI MS: $m / z$ $313.8(\mathrm{M}-\mathrm{H})^{+}$. Elemental analysis of $\mathrm{C}_{15} \mathrm{H}_{10} \mathrm{~N}_{3} \mathrm{OSCl}$ calcd. (found) \%: C 57.05 (57.07); H 3.19 (3.21); N 13.31 (13.33).

(E)-6-Chloro- $N^{\prime}$-((pyridin-2-yl)methylene)thieno[3,2-c]pyridine-2-carbohydrazide (7b): Off white solid; Yield: 120 mg, $86.2 \%$; m.p. $295-298{ }^{\circ} \mathrm{C}$; IR (KBr, $\left.v_{\max }, \mathrm{cm}^{-1}\right): 3081$ (-CO-NH str.), 1667 (-C=O), $1554(-\mathrm{C}=\mathrm{N}$ str. $), 1376(\mathrm{C}=\mathrm{C}$ str. $)$, 1260 (-C-N str.), 638 (C-Cl). ${ }^{1} \mathrm{H}$ NMR (500 MHz, DMSO-d $)$ : $\delta 12.47(* 12.40, \mathrm{~s}, 1 \mathrm{H}), 9.17(\mathrm{~s}, 1 \mathrm{H}), 8.65(\mathrm{~d}, J=4.5 \mathrm{~Hz}, 1 \mathrm{H})$, $8.57(* 8.49, \mathrm{~s}, 1 \mathrm{H}), 8.35(\mathrm{~s}, 1 \mathrm{H}), 8.23(\mathrm{~s}, 1 \mathrm{H}), 7.98-7.94(\mathrm{~m}, 2 \mathrm{H})$, 7.47 (brs, $1 \mathrm{H})$; ESI MS: $m / z, 314.3(\mathrm{M}-\mathrm{H})^{+}$. Elemental analysis of $\mathrm{C}_{14} \mathrm{H}_{9} \mathrm{~N}_{4} \mathrm{OSCl}$ calcd. (found) \%: C 53.08 (53.05); $\mathrm{H} 2.86$ (2.85); N 17.69 (17.66).

(E)-6-Chloro- $N^{\prime}$-((pyridin-3-yl)methylene)thieno[3,2-c]pyridine-2-carbohydrazide (7c): Light yellow solid; Yield: $115 \mathrm{mg}, 82.6 \%$; m.p.: $310-313{ }^{\circ} \mathrm{C}$; IR $\left(\mathrm{KBr}, v_{\max }, \mathrm{cm}^{-1}\right): 3078$ (-CO-NH str.), 1665 (-C=O), $1552(-\mathrm{C}=\mathrm{N}$ str. $), 1374(\mathrm{C}=\mathrm{C}$ str. $)$, 1260 (-C-N str.), 636 (C-Cl). ${ }^{1} \mathrm{H}$ NMR (500 MHz, DMSO-d $)$ : $\delta 12.46$ (* 12.37, s, 1H), 9.17 (s, 1H), 9.01 (*8.90, s, 1H), 8.66 $(\mathrm{s}, 1 \mathrm{H}), 8.54(\mathrm{~d}, J=10.0 \mathrm{~Hz}, 1 \mathrm{H}), 8.37(\mathrm{~d}, J=6.0 \mathrm{~Hz}, 1 \mathrm{H}), 8.38-$ 8.17 (m, 2H), 7.53 (brs, 1H); ESI MS: $m / z, 316.7(\mathrm{M}+\mathrm{H})^{+}$. Elemental analysis of $\mathrm{C}_{14} \mathrm{H}_{9} \mathrm{~N}_{4} \mathrm{OSCl}$ calcd. (found)\%: $\mathrm{C} 53.08$ (53.04); H 2.86 (2.88); N 17.69 (17.66).

(E)-6-Chloro- $N^{\prime}$-((pyridin-4-yl)methylene)thieno[3,2-c]pyridine-2-carbohydrazide (7d): Off white solid; Yield: 119 mg, $85.5 \%$; m.p. $357-359{ }^{\circ} \mathrm{C}$; IR (KBr, $\left.v_{\max }, \mathrm{cm}^{-1}\right)$ : 3081 
<smiles>CCOC(=O)c1cc2cnc(Cl)cc2s1</smiles><smiles>CC(C)C</smiles>

5

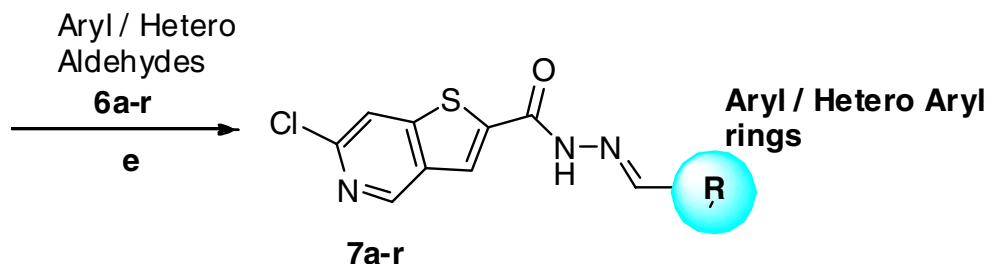

List of $\mathbf{R}=$ Aryl / Hetero Aryl aldehydes

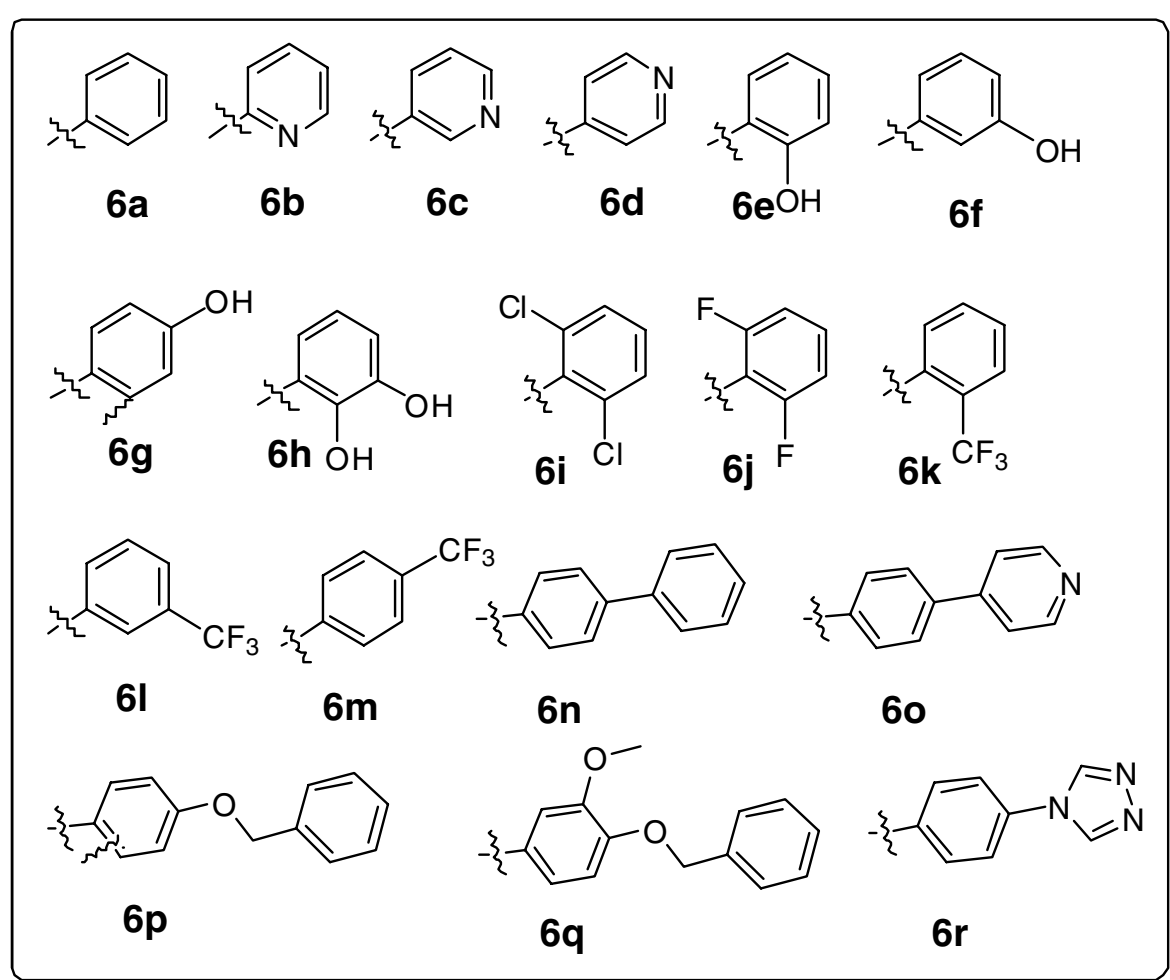

Reaction conditions: a) $\mathrm{NaBH}_{4}$, THF: $\mathrm{MeOH}, 0{ }^{\circ} \mathrm{C}$ to room temperature, $4 \mathrm{~h}$; b) $\mathrm{MnO}_{2}$, methanol, $60{ }^{\circ} \mathrm{C}, 18 \mathrm{~h}$; c) ethylmercapto acetate, triethylamine, dichloromethane, $50{ }^{\circ} \mathrm{C}$ for $8 \mathrm{~h}$; d) hydrazine-hydrate, ethanol, $80^{\circ} \mathrm{C}, 18 \mathrm{~h}$; e) aromatic/hetro aromatic aldehydes $6 \mathrm{a}-\mathrm{r}$, ethyl alcohol, $80{ }^{\circ} \mathrm{C}, 30 \mathrm{~min}$

Scheme-I: 6-Chlorothieno[3,2-c]pyridine-2-carbohydrazide derivatives (7a-r)

(-CO-NH str.),1667 (-C=O), 1588 (-CH=CH-CO, $\alpha, \beta$-unsaturated str.), $1376(\mathrm{C}=\mathrm{C}$ str. $) .{ }^{1} \mathrm{H}$ NMR $\left(500 \mathrm{MHz}, \mathrm{DMSO}-d_{6}\right): \delta$ $10.23(* 10.09, \mathrm{~s}, 1 \mathrm{H}), 9.18(* 9.03, \mathrm{~s}, 1 \mathrm{H}), 8.89(\mathrm{~d}, J=4.5 \mathrm{~Hz}$, $1 \mathrm{H}), 8.76-8.66(\mathrm{~m}, 1 \mathrm{H}), 8.37(* 8.29, \mathrm{~s}, 1 \mathrm{H}), 8.12(\mathrm{~s}, 1 \mathrm{H}), 7.8$ $1(\mathrm{~d}, J=4.5 \mathrm{~Hz}, 2 \mathrm{H})$; ESI MS: $\mathrm{m} / z 317.08(\mathrm{M}+\mathrm{H})^{+}$. Elemental analysis of $\mathrm{C}_{14} \mathrm{H}_{9} \mathrm{~N}_{4} \mathrm{OSCl}$, calcd. (found)\%: C 53.08 (53.10); H 2.86 (2.89); N 17.69 (17.70).

(E)- $N^{\prime}$-(2-Hydroxybenzylidene)-6-chlorothieno[3,2-c]pyridine-2-carbohydrazide (7e): White solid; Yield: $120 \mathrm{mg}$, $82.3 \%$; m.p. $289-282^{\circ} \mathrm{C}$; IR ( $\left.\mathrm{KBr}, v_{\max }, \mathrm{cm}^{-1}\right): 3330$ (-OH str.), 3218 (-CO-NH str.), 1666 (-C=O str.), 1588 ( $-\mathrm{C}=\mathrm{N}$ str.), 1433 $(-\mathrm{C}=\mathrm{C}$ str. $), 1378$ (-C-N str.), 615 (C-Cl). ${ }^{1} \mathrm{H}$ NMR $(500 \mathrm{MHz}$, DMSO- $\left.d_{6}\right): \delta 12.52(* 12.11, \mathrm{~s}, 1 \mathrm{H}), 9.70(* 9.65, \mathrm{~s}, 1 \mathrm{H}), 9.16$ (s, 1H), 8.53 (s, 1H), 8.39-8.34 (m, 1H), 8.09 (s, 1H), 7.33- $7.22(\mathrm{~m}, 3 \mathrm{H}), 6.88(\mathrm{t}, J=10.0 \mathrm{~Hz}, 1 \mathrm{H})$; ESI MS: $m / z, 317.08$ $(\mathrm{M}+\mathrm{H})^{+}$. Elemental analysis of $\mathrm{C}_{15} \mathrm{H}_{10} \mathrm{~N}_{3} \mathrm{O}_{2} \mathrm{SCl}$ calcd. (found) \%: C 54.32 (54.30); H 3.04 (3.01); N 12.67 (12.66).

(E)- $N^{\prime}$-(3-Hydroxybenzylidene)-6-chlorothieno[3,2-c]pyridine-2-carbohydrazide (7f): Off white solid;Yield: 124 mg, $85.1 \%$; m.p. $290-293^{\circ} \mathrm{C}$; IR (KBr, $\left.v_{\max }, \mathrm{cm}^{-1}\right): 3322(-\mathrm{OH}$ str.), 3220 (-CO-NH str.), 1668 ( $-\mathrm{C}=\mathrm{O}$ str. $), 1581(-\mathrm{C}=\mathrm{N}$ str. $)$, 1436 (-C=C str.), 1374 (-C-N str.), 621 (C-Cl) . ${ }^{1} \mathrm{H}$ NMR (500 MHz, DMSO- $\left.d_{6}\right): \delta 12.26(* 12.17, \mathrm{~s}, 1 \mathrm{H}), 9.71(* 9.69, \mathrm{~s}, 1 \mathrm{H})$, $9.15(\mathrm{~d}, J=8.5 \mathrm{~Hz}, 1 \mathrm{H}), 8.53(* 8.09, \mathrm{~s}, 1 \mathrm{H}), 8.38-8.31(\mathrm{~m}$, 2H), 7.31-7.13 (m, 3H), $6.87(\mathrm{t}, J=10.0 \mathrm{~Hz}, 1 \mathrm{H})$; ESI MS: $m / z, 317.08(\mathrm{M}+\mathrm{H})^{+}$. Elemental analysis of $\mathrm{C}_{15} \mathrm{H}_{10} \mathrm{~N}_{3} \mathrm{O}_{2} \mathrm{SCl}$ calcd. (found) \%: C 54.28 (54.30); H 3.02 (3.01); N 12.64 (12.62). 
(E)- $N^{\prime}$-(4-Hydroxybenzylidene)-6-chlorothieno[3,2-c]pyridine-2-carbohydrazide (7g): White solid; Yield: $119 \mathrm{mg}$, $81.6 \%$; m.p. $319-321{ }^{\circ} \mathrm{C}$; IR ( $\left.\mathrm{KBr}, v_{\max }, \mathrm{cm}^{-1}\right)$ : 3314 (-OH str.), 3234(-CO-NH str.), 1666 (-C=O str.), 1576 (-C=N str.), 1428 (-C=C str.), 1370 (-C-N str.), 628 (C-Cl). ${ }^{1} \mathrm{H}$ NMR (500 MHz, DMSO- $\left.d_{6}\right): \delta 12.28(* 12.10, \mathrm{~s}, 1 \mathrm{H}), 9.76(* 9.66, \mathrm{~s}, 1 \mathrm{H}), 9.18$ (d, $J=8.5 \mathrm{~Hz}, 1 \mathrm{H}), 8.50(* 8.10, \mathrm{~s}, 1 \mathrm{H}), 8.38-8.31(\mathrm{~m}, 3 \mathrm{H})$, 7.31-7.13 (m, 4H), $6.86(\mathrm{t}, J=10.0 \mathrm{~Hz}, 1 \mathrm{H})$; ESI MS: $m / z$, $317.08(\mathrm{M}+\mathrm{H})^{+}$. Elemental analysis of $\mathrm{C}_{15} \mathrm{H}_{10} \mathrm{~N}_{3} \mathrm{O}_{2} \mathrm{SCl}$ calcd. (found) \%: C 54.34 (54.29); H 3.06 (3.03); N 12.62 (12.60).

(E)- $N^{\prime}$-(2,3-Dihydroxybenzylidene)-6-chlorothieno[3,2c]pyridine-2-carbohydrazide (7h): Off white solid; Yield: $130 \mathrm{mg}, 85.1 \%$; m.p. $318-321^{\circ} \mathrm{C}$; IR $\left(\mathrm{KBr}, v_{\max }, \mathrm{cm}^{-1}\right)$ : 3343 (-OH str.), 3222(-CO-NH str.), 1668 (-C=O str.), $1572(-\mathrm{C}=\mathrm{N}$ str.), 1417 (-C=C str.), 1366 (-C-N str.), $632(\mathrm{C}-\mathrm{Cl}) .{ }^{1} \mathrm{H}$ NMR $\left(500 \mathrm{MHz}, \mathrm{DMSO}-d_{6}\right): \delta 12.52(* 12.11, \mathrm{~s}, 1 \mathrm{H}), 10.67(* 9.71$, s, $1 \mathrm{H}), 9.33(* 9.11, \mathrm{~s}, 1 \mathrm{H}), 9.16(\mathrm{~s}, 1 \mathrm{H}), 8.65(* 8.54, \mathrm{~s}, 1 \mathrm{H})$, $8.36(\mathrm{~d}, J=4.0 \mathrm{~Hz}, 2 \mathrm{H}), 7.44(* 7.05, \mathrm{~d}, J=6.0 \mathrm{~Hz}, 1 \mathrm{H}), 6.89$ $6.74(\mathrm{~m}, 2 \mathrm{H})$; ESI MS: $m / z, 345.7(\mathrm{M}+\mathrm{H})^{+}$. Elemental analysis of $\mathrm{C}_{15} \mathrm{H}_{10} \mathrm{~N}_{3} \mathrm{O}_{3} \mathrm{SCl}$ calcd. (found) \%: C 51.80 (51.83); H 2.90 (2.89); N 12.08 (12.10).

(E)- $N^{\prime}$-(2,6-Dichlorobenzylidene)-6-chlorothieno[3,2c]pyridine-2-carbohydrazide (7i): Off white solid; Yield: 140 $\mathrm{mg}, 82.8 \%$; m.p. $289-292^{\circ} \mathrm{C}$; IR (KBr, $\left.v_{\max }, \mathrm{cm}^{-1}\right): 3162$ (-CO$\mathrm{NH}$ str. $), 1657$ (-C=O str.), 1575 ( $-\mathrm{C}=\mathrm{N}$ str. $), 1409$ ( $-\mathrm{C}=\mathrm{C}$ str. $)$, 1360 (-C-N str.), 717 (C-Cl). ${ }^{1} \mathrm{H}$ NMR (500 MHz, DMSO- $d_{6}$ ): $\delta 12.56(* 12.42$, s, $1 \mathrm{H}), 9.12$ (br.s, $1 \mathrm{H}), 8.68(* 8.56, \mathrm{~s}, 1 \mathrm{H})$, $8.38(\mathrm{t}, J=10.5 \mathrm{~Hz}, 1 \mathrm{H}), 8.29(\mathrm{~s}, 1 \mathrm{H}), 7.60(\mathrm{~s}, 2 \mathrm{H}), 7.48(\mathrm{~s}, 1 \mathrm{H})$; ESI MS: $m / z, 385.96(\mathrm{M}+\mathrm{H})^{+}$. Elemental analysis of $\mathrm{C}_{15} \mathrm{H}_{8} \mathrm{~N}_{3} \mathrm{OSCl}_{3}$ calcd. (found) \%: C 46.84 (46.80), H 2.10 (2.12), N 10.92 (10.95).

(E)- $N^{\prime}$-(2,6-Difluorobenzylidene)-6-chlorothieno[3,2c]pyridine-2-carbohydrazide (7j): Light yellow solid; Yield: $137 \mathrm{mg}, 88.6 \%$; m.p.: $253-256^{\circ} \mathrm{C}$; IR $\left(\mathrm{KBr}, v_{\max }, \mathrm{cm}^{-1}\right): 3222$ (-CO-NH str.), 1668 (-C=O str.), 1572 ( $-\mathrm{C}=\mathrm{N}$ str. $), 1422(-\mathrm{C}=\mathrm{C}$ str.), 1368 (-C-N str.), 628 (C-Cl). ${ }^{~} \mathrm{H}$ NMR (500 MHz, DMSO$\left.d_{6}\right): \delta 12.45(* 12.31, \mathrm{~s}, 1 \mathrm{H}), 9.15(* 9.10, \mathrm{~s}, 1 \mathrm{H}), 8.64(* 8.60$, $\mathrm{s}, 1 \mathrm{H}), 8.37-8.24(\mathrm{~m}, 2 \mathrm{H}), 7.56(\mathrm{~d}, J=6.4 \mathrm{~Hz}, 1 \mathrm{H}), 7.26(\mathrm{t}, J=$ $9.2 \mathrm{~Hz}, 2 \mathrm{H})$; ESI MS: $\mathrm{m} / z, 352.05(\mathrm{M}+\mathrm{H})^{+}$. Elemental analysis of $\mathrm{C}_{15} \mathrm{H}_{8} \mathrm{~N}_{3} \mathrm{OSClF}_{2}$ calcd. (found) \%: C 51.22 (51.20); H 2.29 (2.27); N 11.95 (11.97).

(E)- $N^{\prime}$-(2-(Trifluoromethyl)benzylidene)-6-chlorothieno[3,2-c]pyridine-2-carbohydrazide (7k): Off white solid; Yield: $139 \mathrm{mg}, 82.4 \%$; m.p.: $292-294^{\circ} \mathrm{C}$; IR (KBr, $\left.v_{\max }, \mathrm{cm}^{-1}\right)$ : 3218 (-CO-NH str.), 1667 (-C=O str.), 1575 ( $-\mathrm{C}=\mathrm{N}$ str.), 1428 $(-\mathrm{C}=\mathrm{C}$ str. $), 1366$ (-C-N str.), $626(\mathrm{C}-\mathrm{Cl}) .{ }^{1} \mathrm{H}$ NMR $(500 \mathrm{MHz}$, DMSO- $\left.d_{6}\right): \delta 12.58(* 12.46, \mathrm{~s}, 1 \mathrm{H}), 9.16(\mathrm{~d}, J=10.5 \mathrm{~Hz}, 1 \mathrm{H})$, $8.84(\mathrm{~s}, 1 \mathrm{H}), 8.56(\mathrm{~d}, J=8.0 \mathrm{~Hz}, 1 \mathrm{H}), 8.38-8.35(\mathrm{~m}, 3 \mathrm{H}), 8.24$ $(\mathrm{d}, J=7.5 \mathrm{~Hz}, 2 \mathrm{H})$; ESI MS: $m / z, 383.60(\mathrm{M}+\mathrm{H})^{+}$. Elemental analysis of $\mathrm{C}_{16} \mathrm{H}_{9} \mathrm{~N}_{3} \mathrm{OSClF}_{3}$ calcd. (found) \%: $\mathrm{C} 50.07$ (50.11); H 2.36 (2.38); N 10.95 (10.97).

(E)- $N^{\prime}$-(3-(Trifluoromethyl)benzylidene)-6-chlorothieno [3,2-c]pyridine-2-carbohydrazide (7l): Off white solid; Yield: $142 \mathrm{mg}, 84.24 \%$; m.p.: $267-269^{\circ} \mathrm{C}$; IR (KBr, $\left.v_{\max }, \mathrm{cm}^{-1}\right): 3165$ (-CO-NH str.), 1663 (-C=O str.), 1573 (-C=N str) $), 1435$ (-C=C str.), 1392 (-C-N str.), 1119 (C-F), 666 (C-Cl). ${ }^{1} \mathrm{H}$ NMR (500 MHz, DMSO- $\left.d_{6}\right): \delta 12.53(* 12.43, \mathrm{~s}, 1 \mathrm{H}), 9.16(\mathrm{~s}, 1 \mathrm{H}), 8.55$ (s, $1 \mathrm{H}), 8.40-8.25$ (m, 3H), 8.12-8.07 (m, 2H), 7.84 (d, $J=6.4 \mathrm{~Hz}$, $1 \mathrm{H})$, 7.76-7.71 (m, 1H); ESI MS: $\mathrm{m} / \mathrm{z}, 384.11(\mathrm{M}+\mathrm{H})^{+}$. Elem- ental analysis of $\mathrm{C}_{16} \mathrm{H}_{9} \mathrm{~N}_{3} \mathrm{OSClF}_{3}$ calcd. (found) \%: C 51.06 (51.08); H 2.28 (2.31); N 11.88 (11.91).

(E)- $N^{\prime}$-(4-(Trifluoromethyl)benzylidene)-6-chlorothieno[3,2-c]pyridine-2-carbohydrazide (7m): Off white solid; Yield: $150 \mathrm{mg}, 88.98 \%$; m.p.: $309-312^{\circ} \mathrm{C}$; $\mathrm{IR}\left(\mathrm{KBr}, v_{\max }, \mathrm{cm}^{-1}\right)$ : 3160 (-CO-NH str.), 1664 (-C=O str.), 1571 ( $-\mathrm{C}=\mathrm{N}$ str.), 1432 (-C=C str.), 1392 (-C-N str.), 1119 (C-F), 666 (C-Cl). ${ }^{1} \mathrm{H}$ NMR (500 MHz, DMSO- $\left.d_{6}\right): \delta 12.51(* 12.40, \mathrm{~s}, 1 \mathrm{H}), 9.18(\mathrm{~s}, 1 \mathrm{H})$, $8.53(\mathrm{~s}, 1 \mathrm{H}), 8.38-8.23(\mathrm{~m}, 3 \mathrm{H}), 8.10-8.05(\mathrm{~m}, 2 \mathrm{H}), 7.85(\mathrm{~d}, J$ $=6.4 \mathrm{~Hz}, 1 \mathrm{H}), 7.74-7.70(\mathrm{~m}, 1 \mathrm{H})$; ESI MS: $m / z, 384.11(\mathrm{M}+\mathrm{H})^{+}$. Elemental analysis of $\mathrm{C}_{16} \mathrm{H}_{9} \mathrm{~N}_{3} \mathrm{OSClF}_{3}$ calcd. (found) $\%$ : C 50.27 (50.24); H 2.34 (2.30); N 10.97 (10.88).

$(E)-N^{\prime}$-([1,1'-Biphenyl]-4-ylmethylene)-6-chlorothieno[3,2-c]pyridine-2-carbohydrazide (7n): Light yellow solid; Yield: $142 \mathrm{mg}, 82.7 \%$; m.p.: $294-296^{\circ} \mathrm{C}$; IR $\left(\mathrm{KBr}, v_{\max }, \mathrm{cm}^{-1}\right)$ : 3155 (-CO-NHstr.), 1654 (-C=O str.), 1573 ( $-\mathrm{C}=\mathrm{N}$ str.), 1400 (-C=C str.), 1340 (-C-N str.), $572(\mathrm{C}-\mathrm{Cl}) .{ }^{1} \mathrm{H}$ NMR $(500 \mathrm{MHz}$, DMSO- $\left.d_{6}\right): \delta 12.36(* 12.28, \mathrm{~s}, 1 \mathrm{H}), 9.18(* 9.15, \mathrm{~s}, 1 \mathrm{H}), 8.56$ $(* 8.52, \mathrm{~s}, 1 \mathrm{H}), 8.38(* 8.23, \mathrm{~s}, 1 \mathrm{H}), 8.36(\mathrm{~s}, 1 \mathrm{H}), 7.96(\mathrm{~d}, J=$ $10.5 \mathrm{~Hz}, 1 \mathrm{H}), 7.87-7.79(\mathrm{~m}, 3 \mathrm{H}), 7.75(\mathrm{~d}, J=9.5 \mathrm{~Hz}, 2 \mathrm{H}), 7.51$ (t, $J=9.0 \mathrm{~Hz}, 2 \mathrm{H}), 7.43(\mathrm{~d}, J=8.5 \mathrm{~Hz}, 1 \mathrm{H})$; ESI MS: $m / z$, $392.10(\mathrm{M}+\mathrm{H})^{+}$. Elemental analysis of $\mathrm{C}_{21} \mathrm{H}_{14} \mathrm{~N}_{3} \mathrm{OSCl}$ calcd. (found) \%: C 64.36 (64.33); H 3.60 (3.59); N 10.72 (10.74).

(E)- $N^{\prime}$-(4-(Pyridin-4-yl)benzylidene)-6-chlorothieno[3,2-c]pyridine-2-carbohydrazide (7o): Off white solid; Yield: $160 \mathrm{mg}, 93 \%$; m.p.: $325-328^{\circ} \mathrm{C}$; IR $\left(\mathrm{KBr}, v_{\max }, \mathrm{cm}^{-1}\right)$ : 3155 (-CO-NH str.), 1661 (-C=O str.), 1585 ( $-\mathrm{C}=\mathrm{N}$ str.), 1506 $(-\mathrm{C}=\mathrm{C}$ str. $), 1398$ (-C-N str.), 671 (C-Cl). ${ }^{1} \mathrm{H}$ NMR $(500 \mathrm{MHz}$, DMSO- $\left.d_{6}\right): \delta 12.42(* 12.33, \mathrm{~s}, 1 \mathrm{H}), 9.17(\mathrm{~d}, J=7.4 \mathrm{~Hz}, 1 \mathrm{H})$, $8.68(\mathrm{~s}, 2 \mathrm{H}), 8.57(* 8.25, \mathrm{~s}, 2 \mathrm{H}), 8.54(* 8.39, \mathrm{~s}, 2 \mathrm{H}), 8.03-$ $7.91(\mathrm{~m}, 3 \mathrm{H}), 7.78(\mathrm{~d}, J=6.0 \mathrm{~Hz}, 2 \mathrm{H})$; ESI MS: $m / 2,392.17$ $(\mathrm{M}+\mathrm{H})^{+}$. Elemental analysis of $\mathrm{C}_{20} \mathrm{H}_{13} \mathrm{~N}_{4} \mathrm{OSCl}$ calcd. (found) \%: C 61.14 (61.11); H 3.34 (3.37); N 14.26 (14.28).

(E)- $N^{\prime}$-(4-(Benzyloxy)benzylidene)-6-chlorothieno[3,2c] pyridine-2-carbohydrazide (7p): Light yellow solid; Yield: $171 \mathrm{mg}, 92.4 \%$; m.p.: $262-266^{\circ} \mathrm{C}$; IR ( $\left.\mathrm{KBr}, v_{\max }, \mathrm{cm}^{-1}\right): 3162$ (-CO-NH str.), 1664 (-C=O), 1575 (-C=N str.), 1506 ( $-\mathrm{C}=\mathrm{C}$ str.), 1389 (C-NC str.), 676 (C-Cl). ${ }^{1} \mathrm{H}$ NMR (500 MHz, DMSO- $\left.d_{6}\right)$ : $\delta 12.20(* 12.11, \mathrm{~s}, 1 \mathrm{H}), 9.15(\mathrm{~d}, J=8.0 \mathrm{~Hz}, 1 \mathrm{H}), 8.53(* 8.41$, $\mathrm{s}, 1 \mathrm{H}), 8.34(\mathrm{~s}, 1 \mathrm{H}), 8.12(\mathrm{~s}, 1 \mathrm{H}), 7.81(\mathrm{~d}, J=8.8 \mathrm{~Hz}, 1 \mathrm{H}), 7.71$ $(\mathrm{d}, J=8.8 \mathrm{~Hz}, 1 \mathrm{H}), 7.48-7.35(\mathrm{~m}, 5 \mathrm{H}), 7.14(\mathrm{t}, J=6.4 \mathrm{~Hz}, 2 \mathrm{H})$; ESIMS: $m / z, 412.7(\mathrm{M}+\mathrm{H})^{+}$. Elemental analysis of $\mathrm{C}_{22} \mathrm{H}_{16} \mathrm{~N}_{3} \mathrm{O}_{2} \mathrm{SCl}$, calcd. (found)\%: C 62.63 (62.65); H 3.82 (3.85); N 9.96 (9.97).

$(E)-N^{\prime}$-(4-(Benzyloxy)-3-methoxybenzylidene)-6-chlorothieno[3,2-c]pyridine-2-carbohydrazide (7q): Off white solid; Yield: $169 \mathrm{mg}, 85.3 \%$; m.p.: $212-216^{\circ} \mathrm{C}$; IR $\left(\mathrm{KBr}, v_{\max }, \mathrm{cm}^{-1}\right)$ : 3154 (-CO-NH str.), 1652 (-C=O str.), 1577 (-C=N str.), 1508 (-C=C str.), 1391 (-C-N str.), 1271 (C-O-C-1), 615 (C-Cl). ${ }^{1} \mathrm{H}$ NMR $\left(500 \mathrm{MHz}, \mathrm{DMSO}-d_{6}\right): \delta 12.21(* 12.18, \mathrm{~s}, 1 \mathrm{H}), 9.14(\mathrm{~s}$, $1 \mathrm{H}), 8.54(* 8.40, \mathrm{~s}, 1 \mathrm{H}), 8.34(\mathrm{~d}, J=9.0 \mathrm{~Hz}, 1 \mathrm{H}), 8.09(\mathrm{~s}, 1 \mathrm{H})$, $7.52(\mathrm{~d}, J=1.5 \mathrm{~Hz}, 1 \mathrm{H}), 7.48(\mathrm{~d}, J=2.0 \mathrm{~Hz}, 2 \mathrm{H}), 7.46-7.35$ (m, 5H), $7.14(\mathrm{~d}, J=10.5 \mathrm{~Hz}, 1 \mathrm{H}), 5.17(* 5.16, \mathrm{~s}, 2 \mathrm{H}), 3.92$ $(* 3.84, \mathrm{~s}, 3 \mathrm{H})$; ESI MS: $m / z, 452.15(\mathrm{M}+\mathrm{H})^{+}$. Elemental analysis of $\mathrm{C}_{23} \mathrm{H}_{18} \mathrm{~N}_{3} \mathrm{O}_{3} \mathrm{SCl}$ calcd. (found)\%: $\mathrm{C} 61.13$ (61.10); $\mathrm{H} 4.01$ (3.99); N 9.30 (9.28).

(E)- $N^{\prime}$-(4-(4H-1,2,4-Triazol-4-yl)benzylidene)-6-chlorothieno[3,2-c]pyridine-2-carbohydrazide (7r): Off white solid; Yield: $152 \mathrm{mg}$, $90.58 \%$; m.p.: $350-353{ }^{\circ} \mathrm{C}$; IR $\left(\mathrm{KBr}, v_{\max }, \mathrm{cm}^{-1}\right)$ : 
3155 (-CO-NH str.), 1661 (-C=O str.), 1585 (-C=N str.), 1506 $(-\mathrm{C}=\mathrm{C}$ str. $), 1398$ (-C-N str. $), 671(\mathrm{C}-\mathrm{Cl}) .{ }^{1} \mathrm{H}$ NMR $(500 \mathrm{MHz}$, DMSO- $\left.d_{6}\right): \delta 12.42(* 12.34, \mathrm{~s}, 1 \mathrm{H}), 9.48(\mathrm{~s}, 1 \mathrm{H}), 9.42(\mathrm{~s}, 1 \mathrm{H})$, 8.57-8.52 (m, 1H), 8.39-8.30 (m, 3H), 8.07-7.95 (m, 4H); ESI MS: $m / z, 383.12(\mathrm{M}+\mathrm{H})^{+}$. Elemental analysis of $\mathrm{C}_{17} \mathrm{H}_{11} \mathrm{~N}_{6} \mathrm{OSCl}$ calcd. (found) \%: C 53.34 (53.37); H 2.90 (2.87); N 21.95 (21.92).

Biological assay: The antibacterial activity experiments were carried out following our previously reported procedure [20,21]. The 6-chlorothieno[3,2-c]pyridine-2-carbohydrazidehydrazone derivatives (7a-r) $(50 \mu \mathrm{g} / \mathrm{mL}$ concentration) were tested in contradiction to Staphylococcus aureus (MTCC 96), Streptococcus pyogenes (MTCC 442) belonging to Gram negative strain and Escherichia coli (MTCC 443), Pseudomonas aeruginosa (MTCC 424) belonging to Gram positive strain, utilizing a standard methodology viz., agar diffusion method where in norfloxacin was used as the reference antibiotic.

\section{RESULTS AND DISCUSSION}

Sodium borohydride reduction of ethyl-4,6-dichloronicotinate (1) in THF:methanol at $0{ }^{\circ} \mathrm{C}$ to room temperature for $4 \mathrm{~h}$ gave (4,6-dichloropyridin-3-yl)methanol (2). $\mathrm{MnO}_{2}$ oxidation of alcohol $2 \mathrm{in}$ methanol at $60^{\circ} \mathrm{C}$ for $18 \mathrm{~h}$ produced 4,6-dichloronicotinaldehyde (3). Condensation of aldehyde 3 with ethyl mercapto acetate in presence of dichloromethane at $50{ }^{\circ} \mathrm{C}$ for $8 \mathrm{~h}$ gave cyclized ethyl ester intermediate 4 . Hydrazinolysis of ethyl ester 4 in ethanol at $80^{\circ} \mathrm{C}$ for $18 \mathrm{~h}$ gave the desired product carbohydrazide [5]. Condensation of carbohydrazide 5 with various aromatic/hetro aromatic aldehydes 6a-r at reflux in ethanol for $30 \mathrm{~min}$ produced 6-chlorothieno[3,2-c]pyridine2-carbohydrazide-hydrazone derivatives (7a-r).

The synthesized hydrazone derivatives of $(E)-N^{\prime}-(2,6-$ dichlorobenzylidene)-6-chlorothieno[3,2-c]pyridine-2carbohydrazide (7a-r) were sufficiently characterized using ${ }^{1} \mathrm{HNMR}$, mass and IR spectroscopic techniques. These hydrazone derivatives were found to exist as a mixture of two rotameric forms in solution $[22,23]$ as indicated by their ${ }^{1} \mathrm{H}$ NMR spectrum. The basis of the ' $E$ ' geometry of $\mathrm{C}=\mathrm{N}$ bond by ${ }^{1} \mathrm{H}$ NMR was established based on the reported literature data $[24,25]$.

As a representative example, structural interpretation of (E)- $N^{\prime}$-(2,6-dichlorobenzylidene)-6-chlorothieno[3,2-c]pyridine-2-carbohydrazide (7i), is described here, the protons resonating at $9.12 \mathrm{ppm}$ appeared as singlet (1H integration) and the proton resonating at $8.29 \mathrm{ppm}$ as singlet $(1 \mathrm{H}$ integration) corresponds to the pyridine ring and proton signal at $7.48 \mathrm{ppm}$ as singlet with $(1 \mathrm{H}$ integration) is assigned to the fused thiophene ring while the protons signals resonating at $12.56(* 12.42$, singlet, $1 \mathrm{H}) \mathrm{ppm}$ and $8.68(* 8.56$, singlet, $1 \mathrm{H})$ ppm corresponds to - $\mathrm{CO}-\mathrm{N} \underline{\mathrm{H}}-$ and $-\mathrm{N}=\mathrm{C} \underline{\mathrm{H}}$ - groups in the scaffold, respectively. Finally, the proton signals resonating at $8.38 \mathrm{ppm}$ (triplet, 1H) and $7.60 \mathrm{ppm}$ (singlet, $2 \mathrm{H}$ ) corresponds to 2,6chlorophenyl ring system. The mass spectrum of compound $7 \mathbf{i}$ showed a molecular ion peak at $m / z, 385.96$ and is in agreement with the desired molecular formula. The distinguished absorption peaks in the region $3162,1657,1575,1360 \mathrm{~cm}^{-1}$ confirms the presence of the desired functional group - $\mathrm{CONH}-$, $-\mathrm{C}=\mathrm{O},-\mathrm{C}=\mathrm{N}$ and $-\mathrm{C}-\mathrm{N}$, respectively as indicated from the IR spectra of compound 7i. Based on the above analytical description, the structure of compound $\mathbf{7 i}$ was determined. Similarly, the remaining compounds 7a-r have been fully characterized as per the above description.

Antibacterial activity: The zone of inhibition values of 6-chlorothieno[3,2-c]pyridine-2-carbohydrazide-hydrazone derivatives (7a-r) obtained from the antibacterial experimentation is given in Table-1. It is evident that compound $7 \mathbf{b}(\mathrm{R}=$ 2-substituted pyridine), 7c ( $\mathrm{R}=3$-substituted pyridine), 7d ( $\mathrm{R}$ $=4$-substituted pyridine), 7o $(\mathrm{R}=4$-phenyl pyridine $), 7 \mathbf{r}(\mathrm{R}=$ 4-phenyl-4H-1,2,4-triazole) exhibited good antibacterial activity. Compounds 7e ( $\mathrm{R}=o$-hydroxyphenyl), $7 \mathbf{f}(\mathrm{R}=m$-hydroxyphenyl), $7 \mathrm{~g}(\mathrm{R}=p$-hydroxyphenyl), $7 \mathbf{h}(\mathrm{R}=o, m$-hydroxyphenyl), 7k ( $\mathrm{R}=o-\mathrm{CF}_{3}$ Phenyl), $7 \mathbf{l}\left(\mathrm{R}=m-\mathrm{CF}_{3}\right.$ phenyl) and $7 \mathbf{m}\left(\mathrm{R}=p-\mathrm{CF}_{3}\right.$ phenyl) moderate antibacterial activity, while compounds $7 \mathbf{a}(\mathrm{R}=$ phenyl), $7 \mathbf{i}(\mathrm{R}=2,6$-dichloro phenyl), $7 \mathbf{j}$ $(\mathrm{R}=2$,6-difluorophenyl) showed weak antibacterial activity. Finally, compounds $7 \mathbf{n}(\mathrm{R}=$ biphenyl), $\mathbf{7 p}(\mathrm{R}=$ (phenoxy-methyl)benzene) and $\mathbf{7 q}(\mathrm{R}=$ (2-methoxyphenoxy)methyl) benzene) did not show any antibacterial activity as they exhibited zero zone of inhibition.

TABLE-1

ZONE OF INHIBITION VALUES OF COMPOUNDS 7a-r $(50 \mu \mathrm{g} / \mathrm{mL})$ FOR THE DETERMINATION OF ANTIBACTERIAL ACTIVITY

\begin{tabular}{ccc|cc}
\hline & \multicolumn{2}{c|}{ Gram-negative } & \multicolumn{2}{c}{ Gram-positive } \\
\cline { 2 - 5 } Compd. No. & $\begin{array}{c}\text { E. } \\
\text { coli }\end{array}$ & $\begin{array}{c}P . \\
\text { aeruginosa }\end{array}$ & $\begin{array}{c}\text { S. } \\
\text { aureus }\end{array}$ & $\begin{array}{c}\text { S. } \\
\text { pyogenes }\end{array}$ \\
\hline $\mathbf{7 a}$ & 8 & 7 & 7 & 6 \\
$\mathbf{7 b}$ & 24 & 19 & 22 & 18 \\
$\mathbf{7 c}$ & 22 & 17 & 21 & 17 \\
$\mathbf{7 d}$ & 21 & 18 & 20 & 17 \\
$\mathbf{7 e}$ & 15 & 11 & 14 & 10 \\
$\mathbf{7 f}$ & 16 & 14 & 16 & 13 \\
$\mathbf{7 g}$ & 16 & 13 & 16 & 12 \\
$\mathbf{7 h}$ & 15 & 14 & 15 & 13 \\
$\mathbf{7 i}$ & 11 & 10 & 11 & 8 \\
$\mathbf{7 j}$ & 10 & 7 & 6 & 5 \\
$\mathbf{7 k}$ & 16 & 15 & 12 & 14 \\
$\mathbf{7 1}$ & 15 & 14 & 14 & 12 \\
$\mathbf{7 m}$ & 16 & 13 & 15 & 11 \\
$\mathbf{7 n}$ & - & - & - & - \\
$\mathbf{7 0}$ & 22 & 18 & 20 & 18 \\
$\mathbf{7 p}$ & - & - & - & - \\
$\mathbf{7 q}$ & - & - & - & - \\
$\mathbf{7 r}$ & 25 & 19 & 23 & 18 \\
*Standard drug & 26 & 21 & 25 & 20 \\
\hline
\end{tabular}

\section{Conclusion}

The synthesis, characterization and antibacterial activity of some new hydrazone derivatives (7a-r) obtained from condensation of 6-chlorothieno[3,2-c]pyridine-2-carbohydrazide and corresponding aromatic and hetroaromatic aldehydes. The antibacterial results of the study demonstrated that compound $\mathbf{7 b}$ ( $\mathrm{R}=2$-substituted pyridine), 7c $(\mathrm{R}=3$-substituted pyridine), 7d ( $\mathrm{R}=4$-substituted pyridine), 7o ( $\mathrm{R}=4$-phenyl pyridine), $7 \mathbf{r}(\mathrm{R}=4$-phenyl-4H-1,2,4-triazole) exhibited good antibacterial activity. Compounds 7e $(\mathrm{R}=o$-hydroxyphenyl), 7f $(\mathrm{R}=m$ hydroxyphenyl), $7 \mathrm{~g}$ ( $\mathrm{R}=p$-hydroxyphenyl), $7 \mathbf{h}(\mathrm{R}=o, m$ hydroxyphenyl), 7k $\left(\mathrm{R}=-o-\mathrm{CF}_{3}\right.$ Phenyl), $7 \mathbf{l}\left(\mathrm{R}=m-\mathrm{CF}_{3}\right.$ phenyl $)$ and $7 \mathbf{m}\left(\mathrm{R}=p-\mathrm{CF}_{3}\right.$ phenyl $)$ moderate antibacterial activity, while the compounds $7 \mathbf{a}(\mathrm{R}=$ phenyl), $7 \mathbf{i}(\mathrm{R}=2,6$-dichloro- 
phenyl), $7 \mathbf{j}$ ( $\mathrm{R}=2$,6-difluorophenyl) showed weak antibacterial activity. Finally, compounds $7 \mathbf{n}(\mathrm{R}=$ biphenyl), $7 \mathbf{p}(\mathrm{R}=$ (phenoxymethyl)benzene) and $\mathbf{7 q}(\mathrm{R}=(2$-methoxyphenoxy)methyl)benzene) did not show any antibacterial activity.

\section{CONFLICT OF INTEREST}

The authors declare that there is no conflict of interests regarding the publication of this article.

\section{REFERENCES}

1. I. Chopra, C. Schofield, M. Everett, A. O'Neill and K. Miller, Lancet Infect. Dis., 8, 133 (2008); https://doi.org/10.1016/s1473-3099(08)70018-5.

2. K.M. Overbye and J.F. Barrett, Drug Discov. Today, 10, 45 (2005); https://doi.org/10.1016/s1359-6446(04)03285-4.

3. C.B. Sangani, D.C. Mungra, M.P. Patel and R.G. Patel, Chin. Chem. Lett., 23, 57 (2012); https://doi.org/10.2478/s11532-011-0041-7.

4. J.S. New, W.L. Christopher, J.P. Yevich, R. Butter, R.S. Schlemmer Jr., C.P. Van der Maelen and J.A. Cipolline, J. Med. Chem., 32, 1147 (1989); https://doi.org/10.1021/jm00126a002.

5. Y. Miyazaki, M. Nakano, H. Sato, A.T. Truesdale, J.D. Stuart, E.N. Nartey, K.E. Hightower and L. Kane-Carson, Bioorg. Med. Chem. Lett., 17, 250 (2007); https://doi.org/10.1002/chin.200719144.

6. A. Khalafi-Nezhad, M.N.S. Rad, H. Mohabatkar, Z. Asrari and B. Hemmateenejad, Bioorg. Med. Chem., 13, 1931 (2005); https://doi.org/10.1016/j.bmc.2005.01.014.

7. J.B.M. Rewinkel, H. Lucas, M.J. Smit, A.B.J. Noach, T.G. van Dinther, A.M.M. Rood, A.J.S.M. Jenneboer and C.A.A. van Boeckel, Bioorg. Med. Chem. Letts., 9, 2837 (1999); https://doi.org/10.1016/s0960-894x(99)00483-7.

8 R.B. Toche and S.N. Chavan, J. Fluoresc., 24, 285 (2014); https://doi.org/10.1007/s10895-013-1313-8.

9. S. Rollas and S.G. Küçükgüzel, Molecules, 12, 1910 (2007); https://doi.org/10.3390/12081910.
10. T. Nasr, S. Bondock and M. Youns, Eur. J. Med. Chem., 76, 539 (2014); https://doi.org/10.1016/j.ejmech.2014.02.026.

11. V. Kumar, G. Basavarajaswamy, M.V. Rai, B. Poojary, V.R. Pai, N. Shruthi and M. Bhat, Bioorg. Med. Chem. Lett., 25, 1420 (2015); https://doi.org/10.1016/j.bmcl.2015.02.043.

12. S. Senkardes, N. Kaushik-Basu, I. Durmaz, D. Manvar, A. Basu, R. Atalay and S.G. Kücükgüzel, Eur. J. Med. Chem., 10, 301 (2016); https://doi.org/10.1016/j.ejmech.2015.10.041.

13. L. Popiolek. Med. Chem. Res., 26, 287 (2017); https://doi.org/10.1007/s00044-016-1756-y.

14. M. Katyal and Y. Dutt, Talanta, 22, 151 (1975); https://doi.org/10.1016/0039-9140(75)80161-5.

15. M. Mohan, M.P. Gupta, L. Chandra and N.K. Jha, Inorg. Chim. Acta, 151, 61 (1988); https://doi.org/10.1016/S0020-1693(00)83485-4.

16. R.B. Singh, P. Jain and R.P. Singh, Talanta, 29, 77 (1982); https://doi.org/10.1016/0039-9140(82)80024-6.

17. Zh.V. Molodykh, B.I. Buzykin and M.P. Kolov, Zh. Obshch. Khim., 60, 222 (1990).

18. P. Vicini, F. Zani, P. Cozzini and I. Doytchinova, Eur. J. Med. Chem., 37, 553 (2002); https://doi.org/10.1016/s0223-5234(02)01378-8.

19. M.G. Mamolo, V. Falagiani, D. Zampieri, L. Vio and E. Banfi, IL Farmaco, 56, 587 (2001); https://doi.org/10.1016/s0014-827x(01)01097-7.

20. N.S. Lakshmana Rao, V.M. Basaveswara Rao and K.R.S. Prasad, World J. Pharm. Res., 6, 914 (2017)

21. N.S. Lakshmana Rao and V.M. Basaveswara Rao, Heterocycl. Lett., 5, 543 (2015)

22. P. Sarbani, J. Chem. Pharm. Res., 2, 387 (2010); https://doi.org/10.1002/ardp.18601510358.

23. R.B. Palmer and N.H. Andersen, Bioorg. Med. Chem. Lett., 6, 2173 (1996); https://doi.org/10.1016/0960-894X(96)00396-4.

24. R. Ivan, E. Felismino, L. Sang, N. Ken-ichi and K. Isao, Bioorg. Med. Chem., 15, 6236 (2007) https://doi.org/10.1016/j.bmc.2007.06.022.

25. G. Palla, G. Predieri and P. Domiano, Tetrahedron, 42, 3649 (1986); https://doi.org/10.1016/s0040-4020(01)87332-4. 